\title{
Sensing Presence (PreSense) Ontology: User Modelling in the Semantic Sensor Web
}

\author{
Amparo-Elizabeth Cano, Aba-Sah Dadzie, Victoria Uren, and Fabio Ciravegna \\ Department of Computer Science, The University of Sheffield, \\ Sheffield, United Kingdom \\ firstinitial.surname@dcs.shef.ac.uk
}

\begin{abstract}
Increasingly, people's digital identities are attached to, and expressed through, their mobile devices. At the same time digital sensors pervade smart environments in which people are immersed. This paper explores different perspectives in which users' modelling features can be expressed through the information obtained by their attached personal sensors. We introduce the PreSense Ontology, which is designed to assign meaning to sensors' observations in terms of user modelling features. We believe that the Sensing Presence (PreSense) Ontology is a first step toward the integration of user modelling and "smart environments". In order to motivate our work we present a scenario and demonstrate how the ontology could be applied in order to enable context-sensitive services.
\end{abstract}

Keywords: Linked data streams, semantic sensor web, user modelling, smart objects.

\section{Introduction}

Digital sensors have pervaded the modern world, and increasingly make up the majority of connected devices, in, e.g., intelligent buildings, traffic lights and in particular in mobile devices. These advances have resulted in "smart" environments, marking an evolution in the generation of information, and the interaction between humans, smart and ordinary devices and sensors. Human-computer interaction now extends to everyday objects attached to the end user or located in their changing environment [1]. Users produce data streams through their mobile devices, wearable and implantable microsensors (e.g., GPS tracklogs, heart rate monitors). These devices now frequently act as the gateway to cyberspace, which is increasingly becoming an extension of the lives of humans in the real, physical world. Therefore, these can provide information regarding a user's physical context (e.g. location, physiological state), in addition to their digital environment (e.g. adding new friends to an online social network, tweeting on an evolving event). This leads to a bond between the user and their mobile devices and sensors, in which the latter act as an extension of the user's identity, providing real-time information that can reveal important user and environmental characteristics.

This provides motivation to explore new techniques for combining current user modelling methods, that depict the digital identity of a given person, with sensor information distributed across the online and physical worlds. 
The contributions of this paper are as follows: we explore different perspectives in which the attachment of sensor data to user models can impact the derivation of tailored services that feed into users' interaction with smart objects and environments, by providing real-time contextualisation. We propose the Sensing Presence (PreSense) ontology as an approach to modelling the attachment of sensor data streams to a user profile, allowing rich, semantic, real-time change in a user's representation. This enables also the integration of observable user features to the linked data cloud [2].

The paper is structured as follows: in section 2 we present the motivation for our work by discussing different perspectives on the use of sensor data for user modelling; in section 2.3 we introduce a scenario that highlights challenges and benefits that the attachment of sensor data to user profiling presents; section 3 discusses existing ontologies that consider sensor data in user modelling; in section 4 we introduce a set of requirements for modelling the attachment of sensor data streams to a user profile; in section 5 we introduce the Sensing Presence Ontology (PreSense). Section 6 describes the application of the ontology in relation to our scenario; finally, section 7 discusses the potential of this work, plans for evaluation, and concludes the paper.

\section{Motivation}

The relevance of users to the Sensor Web has been explored from the perspective of users acting as collective sources of information. Goodchild [3] highlights the relevance of the Social Web in Volunteered Geographic Information, where users have created a mesh of global information. Projects like SensorBase 1 and SensorPedia 2 provide a platform for sharing online sensor information within user communities. However, little attention has been paid to the importance of users' sensors as gateways for personal feature information. This section motivates our work by introducing different perspectives from which users engage with the physical and online worlds through sensor data.

\subsection{Mobility in the Digital Society}

In the past few years, users' online activities, including web browsing, online shopping, and social web media use [45], have served as information sources for user modelling. Further, the emergence of compelling social web platforms (e.g. Facebook ${ }^{3}$, Twitter have encouraged users to proactively participate, shaping their online personae and influencing their perception about how they are viewed by others (a.o., [6]).

Social studies on the adaptation of users to online technologies highlight that users appropriate telecommunications technologies in ways that fit their social groups, life stages, sociability and activities [7]. Since mobility has become a central aspect of the digital society, the introduction of location-aware services in social web platforms for mobile devices has received considerable attention from researchers in recent years. Research in this area includes scenarios for emergency response, tracking, navigation,

\footnotetext{
${ }^{1}$ http: / / sensorbase.org

2 http: / / www . sensorpedia . com

3 http: / / www . facebook. com

4 http://twitter.com
} 
billing and social networking [8]9|10]. Part of the success of these applications is the user's increased dependency on mobile devices; which have become, for some, an indispensable tool. While the use of sensors for registering users' features (e.g. location) has proved to be fundamental in these applications, transient, sensor-based information has, to date, not been considered as an inherent component in user modelling.

\subsection{Sensors and Users' Context}

Sensors refer not only to physical sensor devices but also to values computed as a result of the composition of indirect or abstract measurements derived from multiple, distributed, often heterogeneous data streams [11 12 13]. Such sensors are usually referred to as virtual sensors; they allow the abstraction of data collection away from a fixed set of physical objects. A virtual sensor may define a number of valid sources of information, allowing it to poll for and retrieve information from different sources and at varying levels of granularity.

Following this definition, we consider a web-based sensor as an extension of the concept of virtual sensors, in which the measuring computation involves data streams generated from web resources. A web-based actuator may be regarded as a reactive computation that produces a response to a specified event. E.g. NASA Hurricane 5 on Twitter is a data stream of instantly updated information generated from the continuous monitoring of different devices sensing meteorological conditions for predicting hurricanes and tropical cyclones all over the world.

In the same way, personal data streams may be regarded as gateways reporting relevant information for user modelling. The information embedded in these streams involves different users' context. User context is built on static, stable and dynamic contexts. A user's static and stable contexts represent information about or related to a user that does not, or rarely, changes in time, e.g. the relation between a user and their hometown or work place. A user's dynamic context, in contrast, reflects highly changing information, which is often influenced by the environment in which a user is immersed; this includes, e.g., changes in position, anxiety levels while in a traffic jam.

Advances in intelligent, context-aware systems promote a vision of increasingly autonomous and ubiquitous applications that act on proactive knowledge to provide tailored services to individuals. These smart systems must not only support users in static, pre-defined environments, but also adapt to users' changing context and evolving goals. However, the integration of user context and the user's immersed environmental context, taking into account tempo-spatial restrictions, still requires research. We present next a scenario highlighting the role of sensor data streams in a user profile.

\subsection{Scenario}

Imagine Alice, a Doctor working at a public hospital, and Bob, a Patient suffering from Type II diabetes and obesity. Bob's treatment combines regular insulin injections with a diet plan. His nutritionist works with Alice to monitor how well he follows the plan, his physical activity and the impact both have on his overall health. Periodic reviews will

${ }^{5}$ NASAHurricane: http: / / twitter.com/NASAHurricane 
take this information into account in updating his treatment. Alice must also monitor Bob's blood glucose levels, to determine the suitability of both his diet and medication.

This scenario requires Bob to wear multiple sensors that communicate with Alice over a network. More precisely, it requires the attachment of sensor information to Bob's personal attributes. Given the emergence of sensor-enabled mobile devices, we can imagine that Bob owns a device that connects to the Internet and monitors his location and health [14[15]. In this context, Alice accesses, in real-time, the data generated by Bob's sensors. Should Bob's blood sugar reach a dangerous level, Alice must be able to dispatch emergency assistance to Bob in the most efficient way. Information on his diet is not critical, so is only uploaded periodically.

Let us consider a weekday when Bob is returning to his office after inspecting a construction site with a client. His sensors have recorded higher than usual physical activity and that he missed his usual mid-morning snack. His smartphone warns him of the danger of his blood glucose levels dropping too low. Since it is close to lunchtime his nutrition monitor (NutrApp) polls for suitable eateries between his current location and his office (see Fig. 11A). It also checks Bob's online social network for recommendations by friends he often eats out with. The NutrApp polls for the ingredients of meals and portion sizes from virtual sensors, and determines suitability by matching with the requirements of his diet plan. Time to cook is also important - his calendar has posted a reminder about an early afternoon meeting he must prepare for (Fig.11B). By merging online information with GPS the NutrApp will try to locate members of Bob's social network in the neighbourhood, whose calendars or status information show they are available - if any are found Bob will receive a suggestion to invite them to join him.

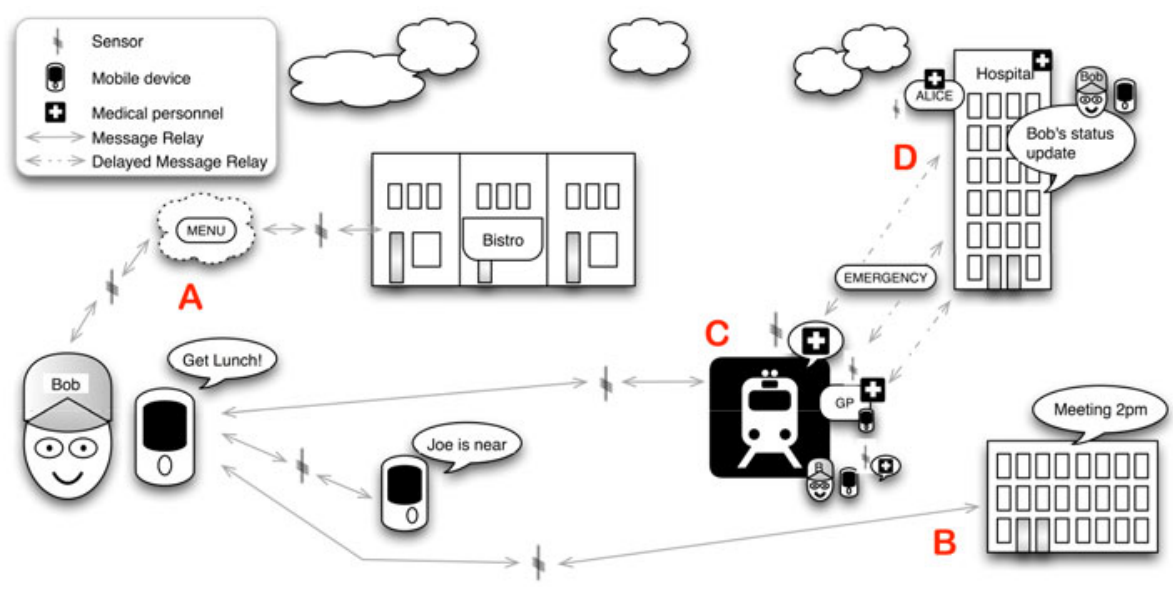

Fig. 1. Schematic for the PreSense scenario, illustrating the exchange of data streams between sensors, and the interaction between human actors and other entities as a result

To illustrate an emergency in which situational context is communicated to external actors via sensors, let us consider what happens if Bob ignores the alerts he receives to stop for lunch, because he forgot to carry his medication. He decides to return directly to 
his office, 45 minutes away via the subway. However, due to a signal failure Bob's train is held stationary just outside a station 1 hour later (Fig. 11C). The stressful situation, combined with more exercise than normal and the time since his last meal, result in a large drop in his blood sugar. Without the sensors recording Bob's blood sugar he may not recognise symptoms of hypoglycaemia till they become severe. In our scenario his smartphone warns him to consume food or drink with high sugar content urgently. His sensors attempt to warn Alice when his blood sugar reaches a critical threshold; however with the train stuck in a tunnel Bob's sensors are unable to connect to Alice's.

Bob's sensors also attempt to locate nearby resources that can help to alleviate his symptoms. A General Practitioner (GP) in the next carriage receives the emergency alert. Virtual sensors apply a context-sensitive filter to Bob's medical information (some of which is held on his personal devices). Another virtual sensor calculates Bob's location using GPS and a schematic of the train and transmits this to the GP's mobile device over a (local) wireless network. The GP locates Bob, and armed with the information needed to attend to the semi-conscious patient, successfully handles the emergency.

When the train exits the tunnel the delayed emergency alert is relayed to Alice (Fig.11D), with a timestamp that indicates that it has now expired. An update with more current, valid information on Bob's status is also relayed to Alice over the Internet.

To be effective, this scenario implies the need to connect different information streaming sources in time- and location-constrained situations, via (context-sensitive) virtual sensors. Particularly, it illustrates the demands of attaching streaming information to real world entities such as people - the GP must be able to identify the patient via sensor stream ownership. Wireless networks also play a role in information exchange; in the emergency situation this is how the virtual and physical sensors communicate.

\section{Related Work}

Ontologies for user modelling follow two paradigms: standardisation- and mediationbased modelling [16]. The first is based on a top-down approach in which ontologies for user modelling are designed to be domain-independent (top-level ontologies), or still high-level but domain-specific (upper ontologies) in order to be reusable by multiple systems. The second is a bottom-up approach which proposes an integrated user model for a specific goal within a specific context [17].

The Friend of a Friend (FOAF) ontology 6 is a top level ontology that models generic information about a user, including their name, social graph, interests and location. However, current FOAF profiling is based on the static representation of, in some cases, highly changing data, such as the temporal location of a user, or their current position in the world (à la foursquare7). Since many of these highly changing user properties can be observed through sensors, different ontologies for considering sensor data in user modelling have emerged. The Service-Oriented Context-Aware Middleware (SOCAM) ontology [18] is an upper ontology which introduces concepts like Activity, Location, ComputationalEntity and Time under the umbrella concept of ContextEntity. Although it models sensors using the Device concept,

\footnotetext{
${ }^{6}$ FOAF ontology: http://xmlns.com/foaf/spec

${ }^{7}$ http://www. foursquare.com
} 
it does not provide a link between a sensor and its owner, nor a relation between the sensor's observations and user properties.

The General User Model Ontology (GUMO) is a top-level ontology introduced in 2005 [19]. GUMO is based on the User Mark-up Language (UserML) [20] and considers dimensions including personality, demographics, emotional and physiological state. The use of sensors in GUMO is considered particularly for users' physiological state; Heckman et al. [19] suggest the use of wearable bio-sensors to register users' body conditions such as pupil dilation and blood pressure. Although they consider the use of UbisWorld 8 for integrating users in ubiquitous environments, there is no clear definition of the way in which a sensor's relationship with a user's properties could be addressed.

The Ontonym ontologies [21] are a collection of seven upper ontologies for pervasive computing. including the Sensor, Device and Person ontologies. They are designed to allow the definition of ownership between a Sensor and a Person through the Device class's owns and ownedBy properties. However, Ontonym requires the definition of a new ontology to map each sensor observation to user properties. For example, to add a relationship between a user's mobile device GPS's location observations and the user's location, the Location ontology is defined to declare the LocatableEntity and LocatableFeature classes (and associated properties). Ontonym is, to the best of our knowledge the only user modelling ontology available online.

Work done in sensor data integration into ontology-based user modelling following a bottom-up approach includes the Mobile Ontology-based Reasoning and Feedback System (MORF) [22], which defines a set of domain-specific ontologies which include classes such as Patient, Doctor and HeartRateSensorData. Their model allows monitoring and transmitting a patient's data through a mobile device. However, restrictions due to domain-specific design prevent MORF and other such bottom-up ontologies from being extensible to generic user modelling.

Relevant components of standard ontologies are discussed in the requirements identified in section 4 and revisited in 5.4 where we assess the extent to which these are met.

\section{Requirements}

The scenario presented in section 2.3 highlights not only the relevance of the identification of sensors and their observations as meaningful web resources, but also the importance of addressing the generated data streams as users' feature properties. In this section, we identify requirements for associating sensor data to user modelling.

Identification and Addressability: To uniquely identify and dereference sensor resources. In our scenario, Alice should be able to identify Bob's sensors, as well as the potential relations among these sensors. For example, by exposing Bob's physical activity and sugar levels, through the definition of his pedometer, as well as his glucose sensor as web resources, health care services could react in a contingency situation, in which external entities such as nearby emergency medical services could respond according to Bob's physical location (see section 6.2).

\footnotetext{
${ }^{8}$ UbisWorld can be tested at: http: / / www . ubisworld. org

${ }^{9}$ Ontonym ontologies: http: / / ontonym.org
} 
Sensor Ownership and Provenance: To establish the sources of information, including entities and processes involved in the generation of measurements from observed stimuli. Provenance in sensor data is crucial for assessing trust judgements on information. An Entity, in particular a Person, should be able to address a sensor as its own - Bob, for instance, should be able to associate sensors with himself. Given a sensor data stream, it should be possible to access the sensor publishing the given stream and identify the sensor's owner. In the scenario, Alice and the GP should be able to identify the streams they are consuming as Bob's.

Association of Sensor Data and Profile Information: To map explicitly, a user's property characterised by a stimulus with the sensor that observes this stimulus. In the scenario, Alice must be able to associate Bob's (continuously changing) location with, e.g., Bob's current_location property, observed by his GPS.

Privacy in Data Streams: To consider how identity information should be exposed and to whom: (1) The consumer of a data stream should be guaranteed that no other service has impersonated the sender; (2) The owner of a data stream should be able to establish authentication methods so only authorised consumers have access to it. E.g., besides Bob, Alice should be authorised to access Bob's health information, as well as the closest emergency doctor who treats Bob at the scene (the latter will have access to a filtered view).

Sensor Data Expiration: To enable a data stream to declare an estimation of the period of time in which its data should be considered valuable. In our scenario, Alice must be able to tell if the received information is still valid, e.g., Alice must know the latest (valid) position of Bob and the time beyond which it is no longer valid.

Interaction with Smart Entities: To allow the representation of collective stimuli in which different entities, including the user, are involved. With collective stimuli we refer to the aggregation of common detectable changes in observable properties. E.g., Bob's location-based proximity social graph is a property derived from the collective stimulus of being located in the vicinity of Bob, within a radius of $5 \mathrm{~km}$.

Integrate Physical and Virtual Presence Stimuli: To identify and incorporate virtual and physical stimuli as part of a user's presence. This integration would bridge the user's physical and online personae. In the scenario, the NutrApp would make use of Bob's online social network to obtain the references of those entities to be monitored for physical presence proximity.

\section{The Sensing Presence Ontology}

In this section we introduce concepts related to users' presence and present the Sensing Presence (PreSense) Ontology $\sqrt{10}$. It defines key concepts and properties required to describe users' features in terms of virtual sensor observables. PreSense models users as entities whose presence is the aggregation of online and physical properties. It represents sensors' observations for deriving presence properties and particular features of interest, following the Stimulus-Sensor-Observation (SSO) ontology design pattern [23]. Fig. 2] illustrates the structure of the PreSense ontology, focusing on the relationships between its core components.

\footnotetext{
${ }^{10}$ Presense ontology available at: http://purl . org/net/presense/ns
} 


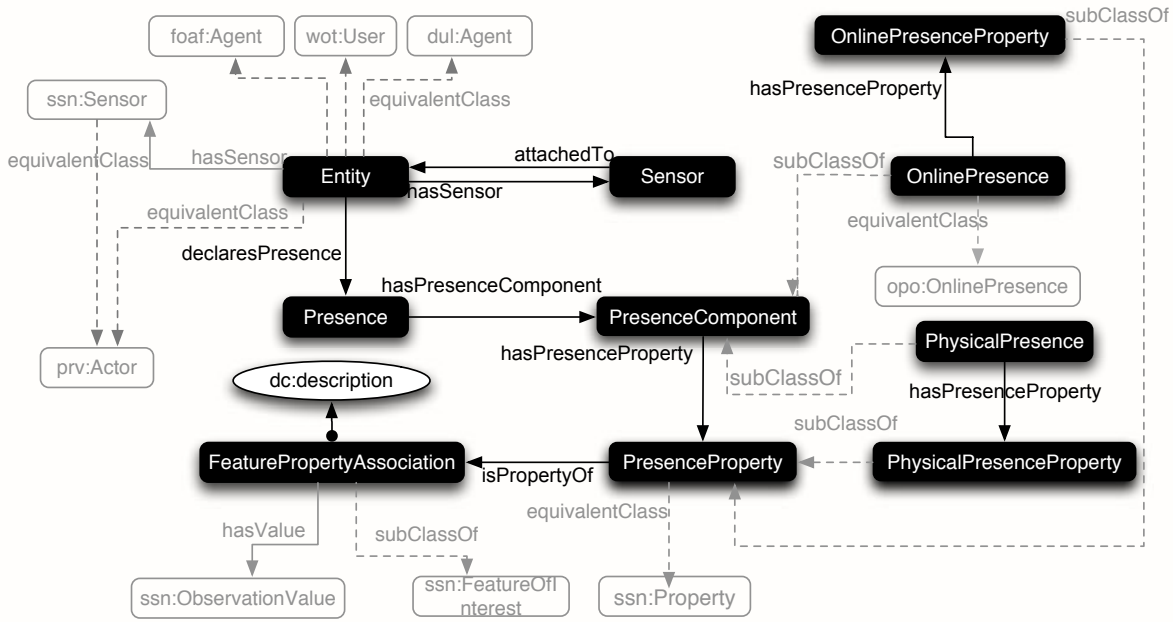

Fig. 2. Sensing Presence Ontology (PreSense) Overview

\subsection{User Modelling Based on Personal Sensors}

Based on the definition of virtual sensors (in section 2.2) and the SSO design pattern [23], we introduce the concept of "personal sensors" to refer to both physical sensor devices and compositions of computations or procedures that measure a user's properties. The information embedded in the data produced by personal sensors includes the users' online and physical presence contexts. By online presence context, we refer to the information provided by the aggregation of personal data streams (e.g. microblog posts, emails, text messages) generated by a user within a window of time. We consider the physical presence context as the abstraction of physical features, measured by sensor devices, regarding a user's state of existence or being present in a place or a thing (e.g. the user's location, body temperature). Both online and physical presence interweave dynamically with a user's surrounding environmental context, which can include other entities like people, places and things (e.g. members of the user's social graph who are close by, or local points of interest - POIs).

\subsection{Imported Ontologies}

Specifications on how to exchange sensor data and their observations have been defined by the Open Geospatial Consortium (OGC). In particular the OGC's Sensor Web Enablement ${ }^{11}$ (SWE) suite is a broad standardisation initiative which comprises models such as the Sensor Model Language 12 (SensorML) and the Observation \& Measurement 13 (O\&M) standards, and services such as the Sensor Observation Service (SOS)

\footnotetext{
11 http: //www. opengeospatial.org/projects/groups/sensorweb

12 http://www.opengeospatial.org/standards/sensorml

13 http: / / www . opengeospatial.org/standards/om
} 
[23]. However, sensor data sharing and discovery expose different challenges involving semantic heterogeneity and integration. The Semantic Sensor Web (SSW) [13] approaches these challenges by providing an ontological platform that defines a machinereadable specification of the conceptualisations that underlie this sensor data.

There are over twelve sensor ontologies [11] for declaring a specification of sensing devices; some include sensors' domain definitions and their relation to observations and measurements. The need for a domain independent and end-to-end model for sensing applications led to the creation of the W3C's Semantic Sensor Network Incubator Group 14 (SSN-XG), who developed the Sensor and Sensor Network (SSN) ontology 15 [24 25]. Taking into account available standards such as the OGC's SWE, the SSN ontology merges sensor-, observation- and system-focused views. The ontology describes sensors following the SSO ontology design pattern [23] and considers spatial provenance properties through the SSN's Deployment module.

Following ongoing research and standardisation efforts, we use the SSN ontology to represent sensors in Presense. Further, we use the Provenance Vocabulary 16 (PRV) [26] to extend provenance-related metadata regarding both sensors and their owners through prv: Actor. For modelling an Entity asserting the ownership of a sensor, we use foaf : Agent. According to the FOAF specification, a foaf : Agent can refer to a person, a group, software or a physical artifact. The Web of Trus 17 (WOT) ontology is used to ensure that the ownership of a sensor cannot be falsified by a third party, thus providing a solid base for valid sensor attachment. PreSense models a user to be equivalent to a wot: User. This equivalence allows a user to assert a digital signature to a web resource, which ensures that: (1) The provenance of the resource cannot be falsified easily; (2) The resource cannot be modified without revoking the provenance of the information.

From the Online Presence Ontology 18 (OPO), we reuse opo: OnlinePresence to model users' online presence properties. Finally, from the Dolce Ultralight Ontology (DUL) 19 we reuse dul: Agent to align existing properties of SSN with a presense: Entity (abbreviated prefix ps: used hereafter), and dul : Situation, in defining the contextual setting of an entity's ps: Presence.

\subsection{Core Components}

Table 1 summarises the requirements fulfilled by each of the core components of the Presense ontology, which we discuss next:

Entity. An entity is modelled to be equivalent to foaf : Agent, wot: User, dul :Agent and prv:Actor. The function of the Entity class is twofold: (1) to describe the identity of an individual (not only persons but entities in general) to whom the sensor data should be attached; and (2) to avoid provenance falsification

\footnotetext{
${ }^{14}$ SSN Incubator Group: http://www.w3 .org/2005/Incubator/ssn/wiki

${ }^{15}$ SSN Ontology: http://purl.oclc.org/NET/ssnx/ssn

${ }^{16}$ Provenance Vocabulary: http://purl.org/net/provenance

${ }^{17}$ WOT Ontology: http://xmlns.com/wot/0.1

${ }^{18}$ Online Presence Ontology: http://online-presence.net/opo/spec

${ }^{19}$ Dolce Ultralight Ontology : http://www. loa-cnr.it/ontologies/DUL. owl
} 
Table 1. Match of core PreSense ontology components to requirements

\begin{tabular}{|c|c|c|c|c|c|c|c|}
\hline & $\begin{array}{l}\text { Ident. \& } \\
\text { Address- } \\
\text { ability of } \\
\text { Sensors }\end{array}$ & $\begin{array}{c}\text { Sensor's } \\
\text { Ownership } \\
\text { \& Prove- } \\
\text { nance }\end{array}$ & $\begin{array}{c}\text { Sensor \& } \\
\text { User } \\
\text { Profile } \\
\text { Assoc. }\end{array}$ & Privacy & $\begin{array}{c}\text { Data } \\
\text { Expiration }\end{array}$ & $\begin{array}{c}\text { Interaction } \\
\text { with Smart } \\
\text { Objects }\end{array}$ & $\begin{array}{c}\text { Integration } \\
\text { of Phys. \& } \\
\text { Virt. Pres. } \\
\text { Stimuli }\end{array}$ \\
\hline Entity & - & - & - & $\circ$ & - & - & - \\
\hline Sensor & - & - & $\bullet$ & - & $\bullet$ & $\bullet$ & - \\
\hline Presence & - & - & - & - & - & $\bullet$ & $\bullet$ \\
\hline PhysicalPresence & - & - & - & - & - & $\bullet$ & $\bullet$ \\
\hline OnlinePresence & - & - & - & - & - & - & $\bullet$ \\
\hline FeaturePropAssoc. & - & - & - & - & - & - & - \\
\hline
\end{tabular}

Legend: • Yes. ○ Limited. - No.

through the use of digital signatures in wot: User. The Entity class considers the property hassensor for attaching a sensor to an entity (its inverse property is attachedTo). Entity is skos : closeMatch with ssn:Platform, which is considered to be an Entity to which a System of sensors is attached. However, SSN considers a Platform to be a dul : Physicalobject which is disjoint with dul : Socialobject.

Sensor. A sensor is defined by the $\mathrm{ssn}$ : Sensor class and refers to a physical object that detects, observes and measures a stimulus. The ps : at tachedTo property is used to assert that a ps: Entity owns this sensor (its inverse property is ps: hassensor). In order to extend provenance metadata of a sensor and its observations, we model the ssn: Sensor to be equivalent to a prv: Actor.

Presence. A Presence refers to the state or fact of existing or being manifest in a place or a thing. We consider that a Presence is an aggregation of an Entity's online and physical manifestations, that occur within a situation or setting. Following DUL, a situation is defined as a "relational context" created by an observer on the basis of a description frame.

Physical Presence. This is the abstraction of the aggregation of physical properties featuring a quality of an entity. These properties are derived by sensors observing physical stimuli. The ps: PhysicalPresence class manifests an entity to be in a state of existing or being present in a place or a thing. These physical presence properties can be broken down into different modules regarding different dimensions in which users' properties can be linked to sensor data.

Online Presence. This is equivalent to opo: OnlinePresence; it refers to the abstraction of the aggregation of online properties featuring a quality of an Entity, e.g., a user. These properties are derived by virtual sensors observing stimuli involving this Entity, e.g., the detection of a user's change of status on a social network site through the ps: OnlinestatusStream.

Feature Property Association. Following the SSO ontology design pattern we introduce this class to bridge a sensor's observed stimulus and the feature that this stimulus characterises in the user model. It is a subclass of ssn: FeatureOfInterest; which being an abstraction of real world phenomena, proxies a stimulus through a quality that can be observed by (an s.sn: Property of) a sensor, and the 
PreSense property describing this quality (i.e., ps : PresenceProperty). The ps: FeaturePropertyAssociation class establishes a relation with the ps: Presence through the property ps : hasPresenceProperty, and by declaring ps: PresenceProperty to be owl : sameAs ssn: Property.

\subsection{Fulfilment of the Requirements}

The PreSense ontology addresses all of the requirements identified in section 4 . Table 2 summarises the differences between the PreSense Ontology and the existing upper ontologies introduced in section 3 .

Table 2. The PreSense Ontology, compared to existing, standard models

\begin{tabular}{|c|c|c|c|c|c|c|c|c|}
\hline & $\begin{array}{c}\text { Ident. \& } \\
\text { Address- } \\
\text { ability of } \\
\text { Sensors }\end{array}$ & $\begin{array}{l}\text { Sensor's } \\
\text { Ownership }\end{array}$ & $\begin{array}{c}\text { Sensor's } \\
\text { Prove- } \\
\text { nance }\end{array}$ & $\begin{array}{c}\text { Sensor \& } \\
\text { User } \\
\text { Profile } \\
\text { Assoc. }\end{array}$ & Privacy & $\begin{array}{c}\text { Data } \\
\text { Expiration }\end{array}$ & $\begin{array}{c}\text { Interaction } \\
\text { with Smart } \\
\text { Objects }\end{array}$ & $\begin{array}{c}\text { Integration } \\
\text { of Phys. \& } \\
\text { Virt. Pres. } \\
\text { Stimuli }\end{array}$ \\
\hline FOAF & - & - & - & - & - & $\circ$ & - & 0 \\
\hline SOCAM & $\circ$ & - & $\circ$ & - & - & 0 & $\bullet$ & $\circ$ \\
\hline GUMO & - & $\bullet$ & - & - & - & $\circ$ & 0 & $\circ$ \\
\hline Ontonym & - & $\bullet$ & $\bullet$ & - & - & $\bullet$ & - & - \\
\hline PreSense & $\bullet$ & • & - & $\bullet$ & $\circ$ & $\bullet$ & • & $\bullet$ \\
\hline
\end{tabular}

Legend: • Yes. ○ Limited. - No.

PreSense uses the SSN: Sensor ontology to model sensors and sensor data. Entity acts as the bridge through which sensor data and profile information can be associated. By reusing the FOAF, WOT and PRV ontologies, entities and sensor ownership can be uniquely identified. The use of WOT partially covers privacy issues. However questions still remain about the correct structure for introducing privacy settings within data streams; we aim to tackle this in future work. Sensor data expiration can be handled using ssn : observationSamplingTime. Presense allows the representation of physical and online presence and their corresponding properties by enabling a bridge between a user's properties and the sensors observing these properties.

\section{Applying PreSense}

This section revisits the scenarios presented in section 2.3 and provides an overview on how to represent different information with the PreSense core ontology.

\subsection{Extending PreSense Core Ontology with Modules}

PreSense modules are extensions to the PreSense core vocabulary that provide additional information regarding a specific type of property. Currently Presense has two modules, the spatial properties module and the health properties module. The spatial property module includes Location, which is a spatial quality of an entity; this 
property is linked to a sensor by the ps: FeaturePropertyAssociation whose value is the observation of, in this case, a GPS sensor. The health properties module considers the Physiologicalstate class and its subclasses (Fig. 3).

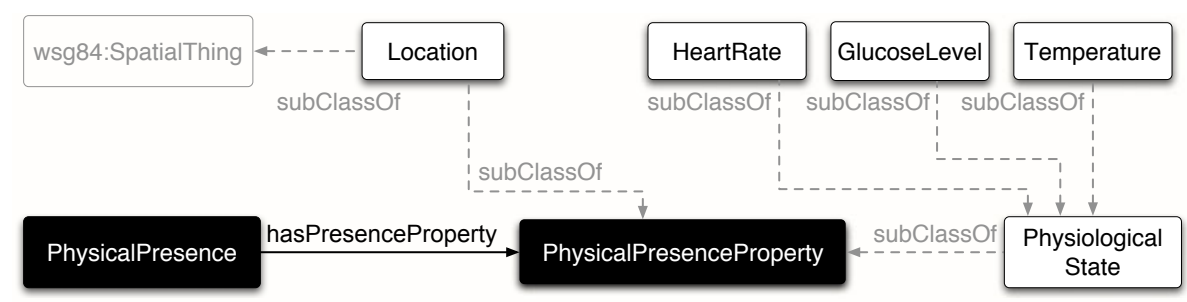

Fig. 3. Presense modules for handling features related to Location and Physiological State

\subsection{Scenario with PreSense}

In this scenario Bob's levels of glucose can be monitored as part of his profile. This could be modelled with the PreSense ontology as:

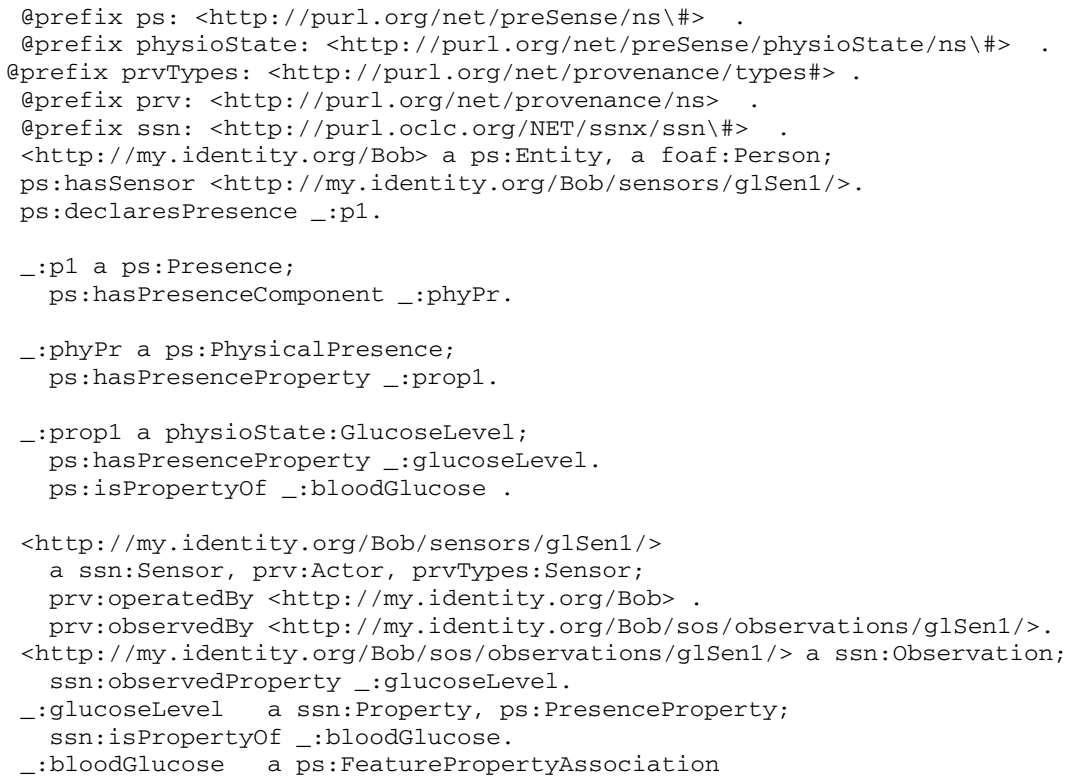

In this example, Bob registers his glucose level measuring sensor ( $g 1$ Sen1) and his physical presence (phy $\mathrm{Pr}$ ). His physical presence considers in this example the health properties module; in particular the glucose level property (glucoseLevel). This 
property corresponds to the property observed by his glSen 1 sensor. This sensor observes changes in his blood sugar levels, (bloodGlucose), which is the feature of interest. This association enables Alice to monitor Bob's sugar levels. Following the URI scheme for linked sensor data proposed by Janowicz et al. [27], Alice could refer to, e.g., http : / / my . identity . org / Bob/sos / observations / glSen1/mgPerdL, which is a reference to all observations gathered by $g 1$ Sen 1 corresponding to the feature of interest bloodGlucose for the observed property, milligrams per decilitre, mgPerdL. In a similar way the PreSense ontology could be applied for registering Bob's heart rate micro-sensor.

The scenario also considers the attachment of virtual sensors to the user's profile. Bob could allow other systems to consume his online status stream (e.g., tweet streams) as:

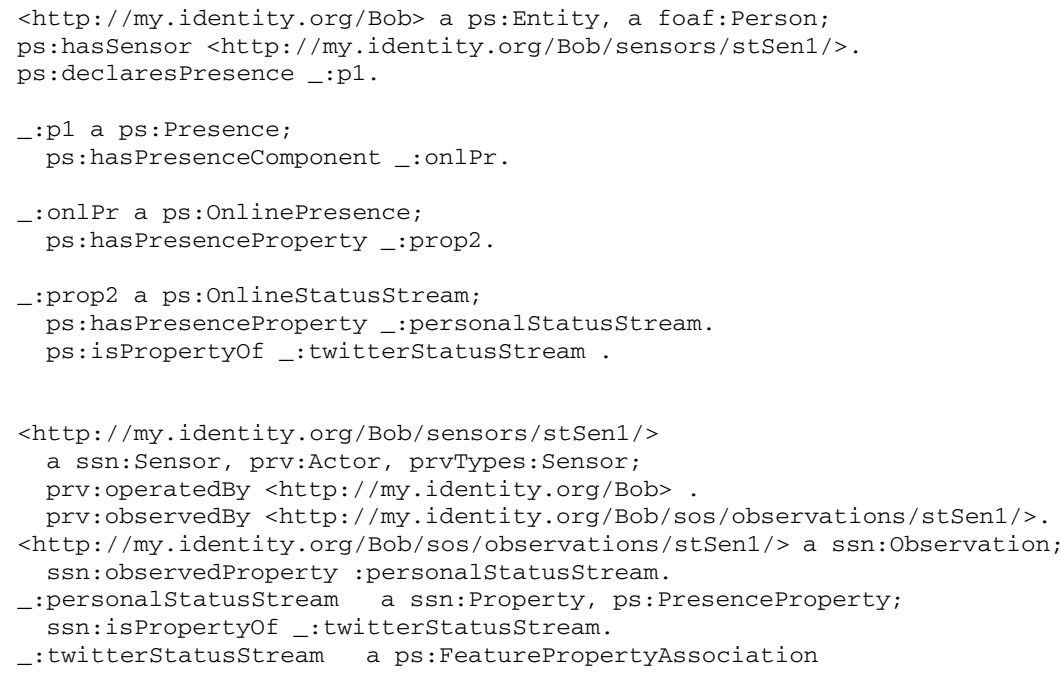

In this case, Bob declares his personal status stream as a property of his online presence onlPr. This property is a proxy for generated contingency tweets on behalf of Bob, and is observed by the virtual sensor stSen 1 . In this case all observations regarding generated tweets could be obtained through http: / / my . identity.org/Bob/sos/observations/stSen1/status. Data derived from his health monitoring devices could trigger an alert when Bob is facing a health contingency situation. This alert could be proxied through Bob's stSen1 sensor; which could alert, e.g., a particular list of Bob's followers in his physical environment about his need for medical attention. They in turn could, on validating the information and its provenance, notify health services about the impending emergency.

\section{Conclusions}

The PreSense ontology is designed to extend people's digital identities through the information obtained by their attached personal sensors. It provides a first step toward 
the integration of user modelling and "smart environments". PreSense distinguishes between the notions of physical presence, e.g., location data obtained from digital sensors, and virtual presence, provided, for instance, by the aggregation of personal data streams, but affords equal status to both. Moreover, PreSense allows the assignment of meaning to sensors' observations in terms of user modelling features.

Future work includes the development of Presense modules addressing interaction with smart entities and environments, by mapping a user's location to that of other nearby entities (NearByPOI and NearByFriends modules). We are also testing the application of the PreSense ontology in real world scenarios, starting with the exploration of new environments and ongoing events.

We are finalising plans for a two-part evaluation of PreSense. The first session will monitor PhysicalPresence in an indoor, smart environment, by tracking the interaction between person Entities to which RFID tags (Sensors) are attached, and fixed objects to which sensor readers will be attached (e.g., a printer - POI), and other sensor-enabled devices (e.g., smart robotic dispensers - Sensor/Entity), in a research laboratory - POI, over a fixed period of time. In this phase we aim to observe and measure physical interaction and FeaturesofInterest in the smart environment as daily working activities take place.

A second evaluation will focus on end users' Onl inePresence, during the Tramlines Festiva 20 in Sheffield at the end of July 2011. Bearing in mind privacy restrictions, we will record only the content of the information exchanged, with associated properties such as Time and Location via the Twitter public stream at selected events and POIs. The information collected will be modelled using PreSense, in order to build a database that maps event type to POIs, and measure the degree of online social interaction during different events. This will allow us to measure how Presense may be used to recommend information to end users based on their profiles and that of their (physical and virtual) social circles both in real-time and over different periods of time.

Acknowledgements. A.E. Cano is funded by CONACyT, grant 175203. A.-S. Dadzie and V. Uren are funded by the European Commission (EC) 7th Framework Programme (FP) project SmartProducts (EC grant ICT-231204). The work reported in this paper was also supported by the EC 7th FP project WeKnowIt (EC grant ICT-215453). The authors thank Gregoire Burel and Matthew Rowe for their valuable comments.

\section{References}

1. Estrin, D., Culler, D., Pister, K., Sukhatme, G.: Connecting the physical world with pervasive networks. IEEE Pervasive Computing 1(1), 59-69 (2002)

2. Bizer, C., Heath, T., Berners-Lee, T.: Linked Data - The Story So Far. International Journal on Semantic Web and Information Systems (2009)

3. Goodchild, M.F.: Citizens as sensors: the world of volunteered geography. Geo. Journal 69, 211-221 (2007)

4. Hogg, T.: Inferring preference correlations from social networks. Electronic Commerce Research and Applications - Special Issue: Social Networks and Web 2.0 9(1), 29-37 (2010)

${ }^{20}$ Tramlines Festival 2011: http: / / www.tramlines.org.uk 
5. Kamis, A., Stern, T., Ladik, D.: A flow-based model of web site intentions when users customize products in business-to-consumer electronic commerce. Information Systems Frontiers 12, 157-168 (2010)

6. Rowe, M.: The credibility of digital identity information on the social web: a user study. In: Proc. 4th ACM Workshop on Information Credibility on the Web, pp. 35-42 (2010)

7. Green, N., Harper, R.H.R., Murtagh, G., Cooper, G.: Configuring the mobile user: Sociological and industry views. Personal and Ubiquitous Computing 5, 146-156 (2001)

8. Hong, D., Chiu, D., Shen, V., Cheung, S., Kafeza, E.: Ubiquitous enterprise service adaptations based on contextual user behavior. Information Systems Frontiers 9, 343-358 (2007)

9. Küpper, A.: Location-Based Services: Fundamentals and Operation. Wiley (2005)

10. Yamada, T., Kaneko, M., Katou, K.: A Mobile Communication Simulation System for Urban Space with User Behavior Scenarios. In: Yang, L.T., Rana, O.F., Di Martino, B., Dongarra, J., et al. (eds.) HPCC 2005. LNCS, vol. 3726, pp. 979-990. Springer, Heidelberg (2005)

11. Compton, M., Henson, C., Neuhaus, H., Lefort, L., Sheth, A.: A survey of the semantic specification of sensors. In: SSN 2009: Proc. 2nd International Workshop on Semantic Sensor Networks at ISWC 2009, pp. 17-32 (2009)

12. Kabadayi, S., Pridgen, A., Julien, C.: Virtual sensors: Abstracting data from physical sensors. In: WoWMoM 2006: Proc. International Symposium on a World of Wireless, Mobile and Multimedia Networks, pp. 587-592 (2006)

13. Sheth, A.P., Henson, C.A., Sahoo, S.S.: Semantic sensor web. IEEE Internet Computing 12(4), 78-83 (2008)

14. Bonato, P.: Wearable sensors and systems. IEEE Engineering in Medicine and Biology Magazine 29(3), 25-36 (2010)

15. Konstantas, D.: An overview of wearable and implantable medical sensors. Yearbook of Medical Informatics 66(9) (2007)

16. Viviani, M., Bennani, N., Egyed-zsigmond, E., Liris, I.: A Survey on User Modeling in MultiApplication Environments. In: Third International Conference on Advances in HumanOriented and Personalized Mechanisms, Technologies and Services (2010)

17. Baumgartner, N., Retschitzegger, W.: A survey of upper ontologies for situation awareness. In: Proc. 4th IASTED International Conference on Knowledge Sharing and Collaborative Engineering, pp. 1-9 (2006)

18. Gu, T., Wang, X.H., Pung, H.K., Zhang, D.Q.: An ontology-based context model in intelligent environments. In: Proc. Communication Networks and Distributed Systems Modeling and Simulation Conference, pp. 270-275 (2004)

19. Heckmann, D., Schwartz, T., Brandherm, B., Schmitz, M., von Wilamowitz-Moellendorff, M.: GUMO - the General User Model Ontology. In: Ardissono, L., Brna, P., Mitrović, A. (eds.) UM 2005. LNCS (LNAI), vol. 3538, pp. 428-432. Springer, Heidelberg (2005)

20. Heckmann, D., Krueger, A.: A user modeling markup language (UserML) for ubiquitous computing. In: Proc. 9th International Conference on User Modeling, pp. 393-397 (2003)

21. Stevenson, G., Knox, S., Dobson, S., Nixon, P.: Ontonym: a collection of upper ontologies for developing pervasive systems. In: CIAO 2009: Proc. 1st Workshop on Context, Information and Ontologies, pp. 1-8 (2009)

22. Benlamri, R., Docksteader, L.: MORF: A mobile health-monitoring platform. IT Professional 12(3), 18-25 (2010)

23. Janowicz, K., Compton, M.: The Stimulus-Sensor-Observation ontology design pattern and its integration into the Semantic Sensor Network ontology. In: SSN 2010: Proc. 3rd International Workshop on Semantic Sensor Networks at ISWC (2010) 
24. Compton, M., Neuhaus, H., Tran, K.N.: Reasoning about sensors and compositions. In: 2nd International Semantic Sensor Networks Workshop (2009)

25. Neuhaus, H., Compton, M.: The semantic sensor network ontology: A generic language to describe sensor assets. In: AGILE Workshop: Challenges in Geospatial Data Harmonisation (2009)

26. Hartig, O.: Provenance information in the web of data. In: LDOW 2009: Proc. Linked Data on the Web Workshop at WWW 2009 (2009)

27. Janowicz, K., Broering, A., Stasch, C., Everding, T.: Towards meaningful URIs for linked sensor data. In: FIS Workshop: Towards Digital Earth: Search, Discover and Share Geospatial Data (2010) 